\title{
Efficient Framework Analysis for Targeted Drug Delivery Based on Internet of Bio-NanoThings
}

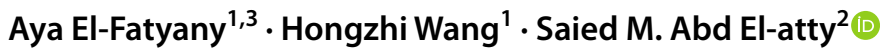

Received: 17 June 2019 / Accepted: 3 April 2021 / Published online: 22 April 2021

(c) King Fahd University of Petroleum \& Minerals 2021

\begin{abstract}
The Internet of Bio-NanoThings (IoBNTs) is a novel paradigm that derives from synthetic biology and advances in nanotechnology for controlling the embedded nanodevices in various medical applications. However, numerous studies have focused on communication efficiency among the nanodevices in a given network, the challenges such as the design and the development of the nanodevices, and the coordination of molecular communication within the wireless body area network (BAN), and the interface connection between the BAN and the Internet are yet to be addressed. Therefore, in this study, we present a framework analysis comprising of the compartmental model, for studying the effects and variances in drug concentration that occur inside intra-body nanonetworks through IoBNT, while taking into account the properties of target cells as well as the ligand-receptor binding mechanism. A performance analysis of the proposed framework for the forward link (i.e., from the Internet to the intra-body nanonetwork) and reverse link (i.e., from the intra-body nanonetwork to the Internet) is presented. The simulation results of the developed framework reveal its ability to enhance the delivery of therapeutic drugs to the target cell while minimizing the side effects in healthy cells.
\end{abstract}

Keywords Nanonetwork $\cdot$ Molecular communication $\cdot$ Internet of bio-nanothings $\cdot$ Target drug delivery

\section{Introduction}

To improve existing technologies and introduce entirely new applications, there has been increasing interest in recent years in the application of nanotechnology concepts, tools, and devices. The main concept of nanotechnology is the manipulation of materials at a particular and molecular level [1]. The application of bionano-technology considered in the present study is the IoBNT. The IoBNT expands upon the Internet of Nanothings (IoNT) by using biologically embedded

Aya El-Fatyany

ayaelfatyany@hit.edu.cn

Hongzhi Wang

wangzh@hit.edu.cn

Saied M. Abd El-atty

sabdelatty@el-eng.menofia.edu.eg

1 School of Computer Science and Technology, Harbin Institute of Technology (HIT), Harbin, China

2 Department of Electronics and Electrical Communications Engineering, Menoufia University, Menouf 32952, Egypt

3 Department of Mathematics, Faculty of Science, Menoufia University, Shebin El-Kom, Egypt computing nanoscale devices. Furthermore, the IoNT and IoBNT stand as novel paradigm-shifting concepts for communication among nanoscale devices interconnected through the Internet [2]. In recent times, the branch of bionano-technology that has attracted the greatest amount of attention is molecular communication (MC) [3]. MC is a new and promising mechanism that uses biochemical signals to achieve an information exchange among naturally and artificially created bio-nano-scale machines over short distances.

MC has been applied in fields such as nanomedicine [4], bioengineering [5], and environmental safety [6]. Other possible applications include intra-body sensing and actuation, intra-body connectivity and control, and environmental control and cleansing [7]. Although the present study focuses on the application of $\mathrm{MC}$ in nanomedicine for a wide range of uses such as targeted drug delivery (TDD) [8] and disease diagnosis, monitoring, and therapy [9], particular focus is given to TDD. A TDD system is a special form of drug delivery system in which the medicament is selectively targeted or delivered only to its site of action or absorption, and not at the target organs, tissues, or cells. TDD seeks to concentrate the medication in the tissues of interest while reducing the relative concentration of the medication in the 
remaining tissues. This improves the efficacy and reduces the side effects. It also reduces the frequency of drug intake and dosage, while maximizing the therapeutic indexes. The idea behind TDD and drug particle transport in the human body, as well as delivery to a specific tissue or organ, can be situated within the context of MC. This means that the transmitter is a drug particle, the information carriers are drug molecules, the communication channel is the blood vessel network, and the receiver is the target tissue [10].

The design of a nanodevice is based on biochemical molecules or existing biological systems. Such nanodevices can be used as analytic and imaging tools, biochemical sensors for monitoring, and tissue engineering for in vivo tissue repair and reengineering, and summarily, for disease diagnosis, monitoring, and therapy [11]. These nanodevices have minute dimensions and their biocompatible nature makes them powerful tools for manipulating biomaterials at the nanoscale level of organisms and systems. Furthermore, these nanodevices are minute; thus, it is easier to control their storage and computational capacity. However, to achieve specific tasks, their cooperation is necessary [12]. In intra-body nanonetworks or a BAN, the number of such nanodevices ranges from a few to millions. Thus, guaranteeing efficient communication among these devices in a given BAN is an important and challenging task. The concept of the IoBNT is derived from the need to enhance a given BAN and ensure a smooth coordination of the communication between the network and the nanodevices connected to other networks, which may be another BAN or a macroscale network such as the Internet. Through the IoBNT system, these nanodevices will execute some simple tasks such as detecting, capturing, storing, releasing, and synthesizing molecules, as well as terminating the functional decomposition [13]. We use the terms nanonetwork and BAN interchangeably in this paper.

\subsection{Motivation and Main Contribution}

The world is now struggling to find solutions using modern information communication technologies such as Internet of Bio-NanoThings (IoBNTs) to treat diseases of the time such as cancer, malignant tumors, and more recently, infectious viruses such as SARS-CoV-2 and COVID 19, by targeting the desired therapeutic medicaments by targeted drug delivery system (TDDS). Therefore, many of researchers have focused their efforts to introduce such solutions, with the help of molecular communications technology. We have introduced some contributions [14-20] in such area. Here, the fundamental goal of our proposed model is to achieve the desired therapeutic medicaments for delivering targeted drug molecules to the diseased cells and hence to reduce the side effects around healthy cells on the same site and thus improved the pharmacokinetic system.
The paper entitled in [21] discussed the body area networks (BANs) communication types. The authors have addressed some of the design challenges that still facing the BAN to become truly ubiquitous for a wide range of applications. The authors in [22] have addressed the area of molecular communication by discussing the molecular architecture layers, applications, design, engineering, and physical modeling. They also have addressed the developing communication protocol challenges and the opportunities for making a large network from bio-nanomachines in future applications. The communication protocol challenge at the physical layer is addressed in [23], however, the authors have designed a protocol approach based on TCP idea. The receiver controls the duration symbol and the detection threshold to prevent inefficient protocol operation for limiting the stored number of the drug molecules in bio-nanomachines. On the other hand, the authors have addressed the congestion problem molecular communication-based diffusion [24]. They proposed a reception model for both illustrating the dynamical behavior of the phenomenon and analyzing the root of it. Furthermore, their proposed model is effective in the range of the short distances. In [25], the authors have proposed a model for molecular communication in respective of molecular network (MN). They have made use of this model to be used in TDD system as well as they showed the design challenges in developing such model. In [26], the authors have addressed the probabilistic approach challenge by proposing an information theoretical model and analyzing the TDD system based on the MC platform. The proposed model provided methods that can be used to define and quantify information exchange among system without needing details knowledge of the system. The security and privacy problems of molecular communication systems have been addressed in [27]. The authors discussed some open issues and challenges for specific directions of potential solutions and highlighted the main advantages and drawbacks.

There are many challenges facing engineering and the realization of a practical IoBNT that we mentioned above. These challenges include the design and the development of the nanodevices, the coordination of molecular communication within the BAN, and the interface connection between the BAN and the Internet. By employing the IoBNT, the previous studies have focused only on communication efficiency among the nanodevices in a given BAN and their application in healthcare delivery systems for separating the drug molecules in the blood vessel channel until they reach the target site. However, these studies ignore the side effects that may occur in healthy cells. Such system may cause the drug to remain in the blood circulatory system for a long time. Further, the authors also have ignored certain diseases that are meant to be targeted with high precision such as tumors, which are highly localized and grow quickly. Therefore, insufficient localization of the target site for tumor cells 
has side effects, it is necessary to find a way that can control the emission of information molecules according to the environment condition [28].

The innovation of this study focuses on the bio-cyber interfaces of the Internet of Biological Nanothings and its employing in a targeted drug delivery system, as shown in Fig. 1. We present an analytical framework based on a compartmental model for the IoBNT while taking into consideration the targeted BANs which represented by the target tissue, and how to deliver therapeutic medicaments to a specific cell (diseased cell) in a way that minimizes side effects. Our main contribution in this paper is as follows:

1. Developing model for the delivery of therapeutic medicaments to the targeted or diseased cells.

2. Modeling a receiver with a specific ligand with a high-affinity binding to the receptors on the targeted cell which improve the effectiveness of therapies and increase the absorption of the drug molecules by the receptors at the surface of the target cell.

3. Evaluating the performance of the proposed framework in the forward and reverse links.

4. Providing the performance comparison with previous work

The rest of this paper is organized as follows. Section 2 presents recent and important related review and research articles. Section 3 presents the system model. In Sect. 4, forward and reverse link based on the compartmental model is introduced; Sect. 5 introduces the extracellular and binding channel. Finally, the numerical results and discussion are presented in Sect. 6. The conclusion of the paper is presented in Sect. 7.

\section{Related Work}

The health monitoring in the form of targeted drug delivery systems (TDDs) and the information technology communication (ICT) in the form of Internet of Biological Nanothings (IoBNT) are combined in order to provide the future healthcare technology or health tech. The paper entitled [29] represents descriptive scenario and system model of the IoBNT which applied it in advanced healthcare delivery system to handle one of the major challenges of the IoBNT, by display architecture and model of bio-cyber interface for connecting biochemical signaling-based bio-nanonetwork to the conventional electromagnetic-based the Internet. The analysis of this paper focused on the system that includes bio-cyber interface and the information propagation network of the blood vessel, which leads to the intra-body nanonetwork location. In [2] presents the architecture model of the IoBNT, which can apply the elimination process of nanodevices through the Internet when it is required. The idea behind this work is providing a deterministic model by allowing the communication interface between the nanotransmitter and the other nanodevices. It focuses on the self-annihilation, which describes that the nanotransmitter is responsible for sending the death command to nanodevices to eliminate the process inside intra-body nanonetwork by using natural cells. The authors [7] describe a new paradigm of the IoBNT, while the author explains the source

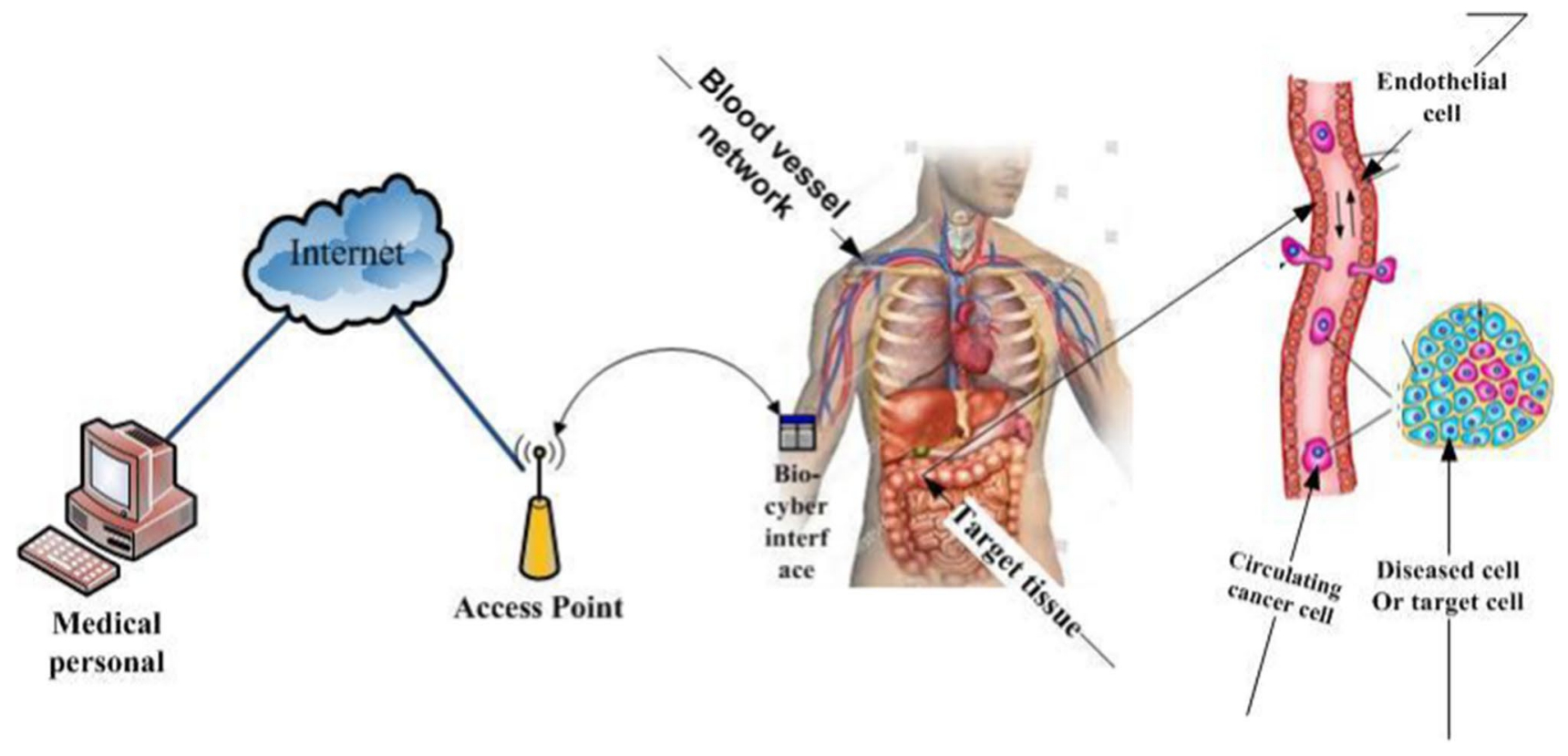

Fig. 1 Illustrative graphic of the system models 
of the IoBNT from a combination of synthetic biology and nanotechnology which the engineering of computing devices can cooperate with biological components. The biological embedded computing devices which depend on biological cells and their functionalities with biochemical domains that are helping on sensing and actuation in the intra-body and environmental control of toxic agents and pollution. This paper also explains that MC is considered as the communication media used by the IoBNT. In normal, the information exchange between cells is based on the emission, transformation, synthesis, propagation, and the receiving of molecules by using biochemical and physical process. In [1] for achieving the effectiveness of TDD at the infected sites, they present MC-based TDD for delivering the drug therapeutic to numerous sites. In this work, the nanotransmitter and nanoreceiver are injected into the targeted body's system blood network and using the compartmental model for delivering the drug molecules to targeted sites. The idea of the effectiveness here is achieved by delivering drug on tissues surface. This effectiveness based on some parameters like the receiver radius, the diffusion characteristics, the total nanotransmitter volume capacity, and the concentration of the enzyme. But, this work not applying the IoBNT model for remotely controlling and delivering the drug molecules to target sites and elimination process. The work titled in [30], the MC paradigm as the information is conveyed through molecules by proposing a model of ADDs to consider the unique, the properties of antibodies, and their possibilities. The idea behind this model to solve some issues of pharmacokinetics models, as mentioned in the state of the art. As this issue is that some diseases like tumors are highly localized and grow quickly and this model does not have enough temporal and spatial accuracy to achieve the efficiency of the ADDs. Although this proposed model solves the issue of the pharmacokinetics model, it is not applying the IoBNT model for remotely controlling the antibodies and spreading the drug molecules inside the target site.

As we mentioned above, most of the studies on the IoBNT focus only on the communication efficiency among the nanodevices in a given targeted BAN and ignore the physical interactions and the characteristics of the protein receptors, i.e., the side effects that may occur in healthy cells. On the other hand, other studies which focus on delivering therapeutic drug efficiently to diseased cells in the targeted site, ignore the efficient communication between nanodevices in this site. Even these studies did not use IoBNT for remotely controlling these nanodevices. In a complementary step with the related works, we present a framework based on compartmental models for targeted drug delivery system with taking into account the receptors binding mechanism. Therefore, the innovation of this work is proposing an analytical framework based on compartmental model while taking into account the binding of ligand-receptor mechanism. We basically present the transmission analysis of the forward and reverse link of the bio-cyber interfaces in IoBNT, take into account targeted BANs, provide efficient communication between nanodevices inside the targeted site. Also, it takes into consideration delivering therapeutic drug specifically to diseased cells in targeted BAN in a way without keeping any effect on healthy cells in the same site. A comparison between the state of the art $[1,2,29,30]$ and the proposed model is illustrated in Table 1

\section{System Model}

Figure 1 illustrates the proposed system model; it consists of the following units, Internet, access point, wireless channel, bio-cyber interface device, blood vessel channel, extracellular channel, and finally, targeted cells. These units based on classical communication are modeled as the time-varying impulse response $h_{1}, h_{2}, h_{3}, h_{4}, h_{5}, h_{6}$, and $h_{7}$, respectively [29]. In this study, we focus on modeling two stages the first stage from the wireless channel to a blood vessel through a bio-cyber interface while the medical personal sends unique binary code orders through the Internet. These orders functions include releasing drug molecules, synthesizing, and sensing proper drug molecules. The Internet of things can be modeled as $h_{1}(t)$ and $h_{2}(t)$. For simplifying the analysis of the system, the effects of the delay and error occurring due to the Internet and access point are ignored. The second stage is from the blood vessel to the targeted cell through the extracellular channel. Our main objective in designing this model is to achieve the desired effects of therapeutic medicament while preventing diseases from progressing quickly by localizing treatment in the specific or target cell through an end-to-end system. While minimizing the side effects in other healthy parts of the body, can occur by fast absorption and elimination of the dose.

Our system model is described as follows. First, starting from the medical personal that sending suitable order information that is transmitted by the access point. Therefore, the communication between the medical personal, Internet, and the access point can be modeled as a wireless channel and its outputs as:

$y^{(n)}(t)=x^{(n)}(t) *\left(h_{1}(t) * h_{2}(t) * h_{3}(t)\right), \quad n=\{f, r\}$

where $x^{(n)}(t)$ denote input signals and * indicates the convolution operator. The direction of the communication is determined by the superscript ${ }^{(\mathbf{n})}$, while ${ }^{(\mathbf{f})}$ indicates the forward link from the medical personnel to the nanodevices in the targeted site and ${ }^{(\mathbf{r})}$ indicates the reverse link from the nanosensors to the medical personnel. The model in Fig. 2 consists of three nanodevices: Nano-R1, Nano-R2, and Nano-R3, and only one nanosensor. The Nano-R1 is injected 
into the vascular system that contains liposome as a nanocarrier and combines tasks such as stimuli response, long circulation, and targeting characteristics [31-33]. This Nano-R1 functions as a transceiver in the blood vessel to propagate the molecules to the targeted site through the extracellular channel after being influenced by external triggers such as $\mathrm{PH}$, light, and enzymes. These external triggers are required to make the Nano-R1 release the drug molecules to the targeted site after it leaves the blood vessel wall through the endothelial gaps on the surface of the wall referred to as the fenestrae. The model depends on two external triggers, $\mathrm{PH}$ and light; when these triggers act on the liposome, it starts to release its contents into the blood vessel.

The Nano-R2 is also a transceiver that synthesizes and emits the specified therapeutic drug. In our work, the Nano-R2 is modeled as the receiver in the nanocarriers with a specific ligand for high-affinity binding to the receptors on the surface of the diseased cells at the targeted site. After the nanocarriers are released by the Nano-R1, they exit the blood vessel wall to reach the target site and bind the receptors on the surface of the Nano-R2 and release the therapeutic drug. The released molecules by the Nano-R1 propagates to the Nano-R2 space wherever the propagation of drug molecules are activated through receptor-mediation or direct diffusion. On the other hand, the Nano-R3 is used to emit molecules to make deathinitiating molecules for artificial cells according to [34]. The process of executing this self-annihilation in nanodevices is represented in [2].

The nanosensor is a bionanosensor device that has high sensitivity for sensing the presence of the nanocarriers in the blood vessel; it responds by emitting its molecules. According to $[34,35]$, the design of the nanosensor is modeled by using the entire cell or sensor molecules, such as in [36]. Biochemical kinetics and its operation are taken into consideration in constructing the mathematical model for the nanosensor. In this study, the drug molecules pass through two channels to get to the target (diseased) cell. The first channel is the cardiovascular system (blood vessel network); in this channel, the propagation of the drug molecules is based on the compartmental model [37] and a conceptual reception space [38]. The second channel is the extracellular channel wherein the propagation of the drug molecules is freely diffused according to diffusion coefficient surrounding a blood vessel in the target tissue or target site. Table 2 describes each part of our proposed model.

\section{Analytical Framework-Based Compartmental Model}

Figure 3 describes the proposed framework-based compartment model which used in this work for the forward and reverse link. The idea behind using compartment model here 
Fig. 2 Illustration of the targeted tissues with diseased cells

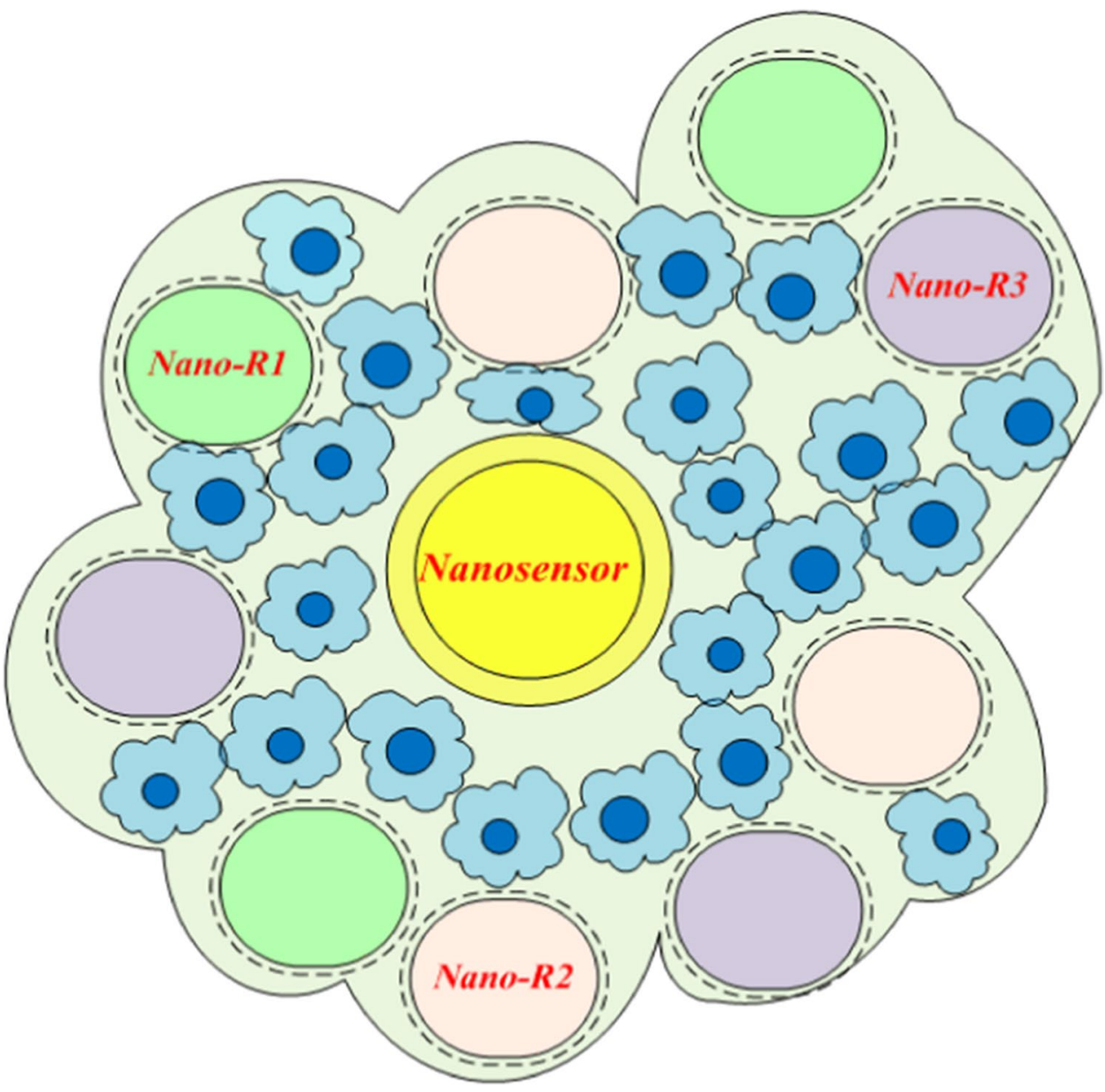

Table 2 System model description

\begin{tabular}{ll}
\hline Parameters & Description \\
\hline$\Phi$ & $\begin{array}{l}\text { Porosity } \\
\text { The concentration of the molecules (Bio- } \\
\text { cyber interface) } \\
m_{0}\end{array}$ \\
$K_{12}$ Kinetic constant \\
$K_{10}$ & Elimination rate \\
$\delta_{0}$ & The concentration of bounding molecules \\
$T$ & Temperature \\
$\xi$ & Number of liposome \\
$r_{R}$ & Reception space \\
$R$ & Distance between transmitter-receiver \\
$K_{12, \mathrm{r}}$ & Forward constant rate \\
$K_{L}$ & Ligand-receptor binding constant \\
$\alpha_{\mathrm{m}}$ & Michaelis-Menten constant \\
$\mathrm{X}$ & Concentration of ATP \\
Nano-R1 & Nanotransmitter (sender) \\
Nano-R2 & Nanoreceiver (receiver) \\
Nano-R3 & Elimination nanodevice \\
\hline
\end{tabular}

is to model the information molecules concentration inside the blood network and accounts the number of the molecules arrived to targeted site and bind to the specific diseased cell after being injected. As represented in Fig. 3, the blood vessel system is the central compartment between bio-cyber interface and the target tissue.

\subsection{Forward Link}

In forwarding link, as shown in Fig. $3 n_{1}(t)$ represents the concentration of information molecules in the blood vessel, while $n_{2}(t)$ is the information molecules concentration in the target tissue. $v_{E}(t)$ is the concentration function of the eliminated molecules over time, along with the elimination rate $k_{10}$. Furthermore, all the molecules affected by the reaction process, phagocytosis, and the adhesion process are absorbed by the non-targeted tissue inside the concentration. The liver is responsible for the elimination process. The first-order constants inside and outside compartment of target tissue for forwarding link are $k_{12} / k_{21}$. The concentration difference between the compartments, the fenestra size through the endothelial cell network, and the diffusion properties of the information molecules are affected by the 


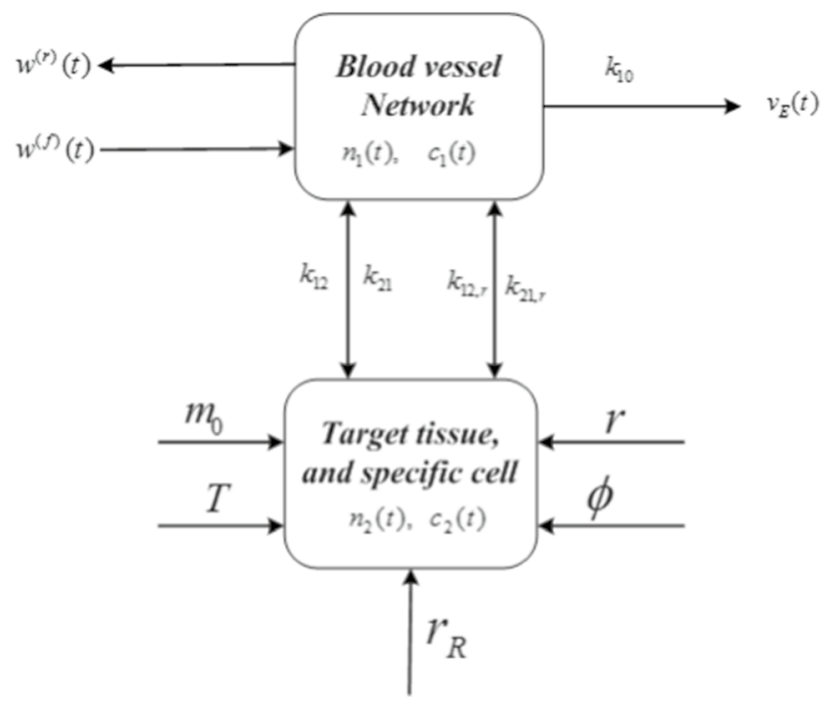

Fig. 3 A compartmental model from the bio-cyber interface to target cell and vice versa

constant rates. According to [13], these constant rates are influenced by these properties. The mathematical equations expatiating on our system for forwarding link in Fig. 3 are represented below:

$\frac{\mathrm{d} n_{1}(t)}{\mathrm{d} t}=-n_{1}(t)\left(k_{12}+k_{10}\right)+k_{21} n_{2}(t)$

$\frac{\mathrm{d} n_{2}(t)}{\mathrm{d} t}=-k_{21} n_{2}(t)+k_{12} n_{1}(t)$

where $n_{1}(0)=w^{(f)}$, and $n_{2}(0)=0$ are the initial conditions in the forward link.

In our system for forwarding link as illustrated in Fig. 1, the medical personal sends signal which is converted to binary code by the bio-cyber interface to stimuli the carriers. In this study, using thermal and light as two stimuli for influencing the contents of the liposomes. Moreover, we used liposomes as nanocarriers for information molecules. According to [39], some nanocarriers, such as the liposomes, require some stimuli such as $\mathrm{PH}$, magnetic field, light intensity, and enzymes to emit their contents. The assumption here is that the molecule emitted by the thermal-responsive liposome is $\beta$, while the molecule emitted by the lightresponsive liposome is $\psi$, according to [29]. The procedure of releasing the molecules carried by the liposomes can be represented as the function of time according to [39]:

$n(t)=\delta_{T}\left(1-e^{-\gamma t}\right)$

The rate of release, $\gamma$ is equal to the rate of the firstorder constant. $\delta_{T}$ denotes the cumulative concentration of the emitted molecules where the release rate of the thermal-responsive liposome is $\gamma_{t}$ and the release rate of the light-responsive liposome is $\gamma_{l}$ as $\gamma_{t}, \gamma_{l} \in \gamma$. The concentration of the emitted molecules function at a constant time can be expressed as the density function as follows:

$\delta(t)=\frac{\mathrm{d}}{\mathrm{d} t}\left[\left(1-e^{-\gamma t}\right) \times \int_{0}^{\infty} \delta(t) d_{t}\right]$

We explain the period of injection as the time $t_{d}$ when the emitted molecules were injected; the release process starts at $t_{R}$. Hence, the output of the bio-cyber interface can be expressed as:

$w^{(f)}=\int_{0}^{t_{d-} t_{R}} \xi \delta(t) \mathrm{d} t$

and

$\delta_{0}=\left.\xi \delta(t)\right|_{t=t_{d}-t_{R}}$

where $\xi$ is the number of liposomes, to ensure that the information is delivered to the Nano-R2 and Nano-R3, we assume that the initial value of $w^{(f)}$ is $n_{0}$ and only one liposome is used.

\subsection{Reverse Link}

When the human body is malfunctioning due to lack of drug molecules delivery or other causes. The nanosensor that synthesizes the emitted drug molecules in the blood vessel sends the information to the IoBNT. Based on the bioluminescent reaction in [40], this reaction produces phosphate group (PP), adenosine monophosphate (AMP), and light $h v$. Thus, the bioluminescence intensity, $I(t)$ equation, including the Luciferase, (LU) and adenosine triphosphate (ATP), can be expressed according to the Michaelis-Menten mechanisms thus:

$I(t)=\frac{\alpha_{l} L X}{X+\alpha_{m}}$

where $X$ and $L$ are the concentration of ATP and LU, respectively, $\alpha_{m}, \alpha_{L}$ is the Michaelis-Menten constant and catalytic reaction constant, respectively. We can obtain the term $L$ from the differential equations in [34] and based on the Hill function in [41, 42].

According to the interaction in the ligand-receptor mechanism which presented in [15], we consider the receptormediated or direct diffusion of information molecules are responsible for activating the transcription factor into the cellular structure that depending on the concentration of the diffusing information molecules in contrast in [43] as the 
transcription factor was oscillatory input. Hence, we can represent the concentration of transcription factor, which describes the proposed bio-cyber interface as:

$m(t) \approx \mu w^{(r)}(t)$

where $w^{(r)}(t)$ is biological signals and $\mu$ is the number of signal conditioning at the cellular structure surface. Subsequently, the concentration rates can be expressed by,

$\frac{\mathrm{d} c_{1}(t)}{\mathrm{d} t}=-\left(k_{12, r}+k_{10}+k_{l}\right) c_{1}(t)+k_{21, r} m_{0} c_{2}(t)$

$\frac{\mathrm{d} c_{2}(t)}{\mathrm{d} t}=-k_{21, r} c_{2}(t)+k_{12, r} c_{1}(t)$

We consider at the instant, $t=0, c_{1}(0)=0$ and $c_{2}(0)=f$ are the initial conditions. Additionally, $m_{0}$ is the total concentration of the molecules emitted by the nanosensor. The term $k_{l}$ is the ligand-receptor binding constant and $k_{12, r} / k_{21, r}$ are the kinetic rate constants. In this work, the molecules follow the Brownian motion for the movement in and out of the compartments and through the blood network. The concentration functions of the molecules $n_{i}(t)$ and $c_{i}(t)$ include the Gaussian noise, according to [44]; specifically in this work, we use the normal distribution, $\mathrm{N}\left(0, \sigma^{2}\right)$ zero-mean, and variance $\sigma^{2}$.

\subsection{Nano-R2 model}

The primary design of the Nano- 2 comprises of a lipopeptide membrane occupied by a target-ligand for targeting. The released drug molecules (liposomes) propagate through the tissue channel (extracellular channel) to the Nano-R2 and binds to the ligand on the surface that is activated by receptor-mediation or direct diffusion. The concentration of the drug molecules $g\left(t, r_{0}\right)$ and the concentration of the active drug molecules received within the conceptual reception space around the Nano-R2 [38] can be expressed by:

$g_{n}\left(t, r_{0}\right) \stackrel{D, r, r_{R}}{\longrightarrow} w_{n}(t)$

where $D$ is the diffusion coefficient of the diffusion channel, $r$ is the distance between the transmitter (Nano-R1) and receiver (Nano-R2) and $r_{R}$ is the radius of the receiver (Nano-R2). The information molecules are formed by a bioluminescent bio-receptor to produce LU [43, 45], which is a receptor protein developed by genetically engineering the LU genes in living cells [46, 47].

We assumed that the Nano-R2 has a position $r_{0}$, based on Eq. (11), in which the concentration of the emitted drug molecules can deduce $r_{0}=r_{R}$ as the drug molecules spotted at the space around Nano-R2. The drug released represents the concentration of the liposome that emits this drug, which is spotted at the immediate region of the neighboring membrane of the Nano-R1. Therefore, supposing the distance between the Nano-R1 and Nano-R2 is large enough to contain the radial solution, the diffusion of the information molecules from the Nano-R1 can be considered as the even distribution of these molecules around Nano-R2 at time $t$ evenly. Thus, we can expatiate on the initial condition as follows:

$g_{R}(0, r)=\frac{1}{4 \pi r} N_{a}$

where $r$ is the distance from the Nano-R1 to the center of the Nano-R2 and $N_{a}$ is the amplitude of $g_{R}(0, r)$. Thus, the concentration of the active drug molecules received in the reception space, $r_{R}$ is represented by the following equation:

$f=\frac{D \cdot N_{a} \cdot\left(r-r_{R}\right)^{2}}{2 \sqrt{\pi}(D t)^{3 / 2} r} e^{\frac{-\left(r_{R}-r\right)^{2}}{4 D t}}$

where $D$ is the diffusion coefficient medium. Therefore, the equations of the compartmental model for the reverse link are represented as:

$w^{(r)}(t)=k_{l} c_{1}(t)$

where $k_{l}$ is the ligand-receptor binding constant.

\section{Extracellular and Binding Channel}

In this section provides the mechanism of the forward link which occurs in the extracellular channel and the binding channel. As illustrated in Fig. 4, the molecules leave the blood vessel and reach the target cell through the extracellular channel. We consider this mechanism as a communication channel engineering problem and thus applying the convolution theory as follows.

\subsection{Extracellular Channel}

The extracellular channel in the proposed model is between the blood vessel tissues and the surface of the target cells, as shown in Fig. 4. It depends on the interstitial pressure between the blood vessel walls and target cells. It is also influenced by the lymphatic flow and the binding of the extracellular channel model (ECM) molecules. As can be seen in Fig. 4, $E(t)$ is the function of the extracellular channel and $m_{E}(t)$ is the function of the concentration of the extracellular molecules. The concentration of the molecules in ECM can be represented as the function of time as follows:

$m_{E}(t)=E(t) * n_{2}(t)$ 


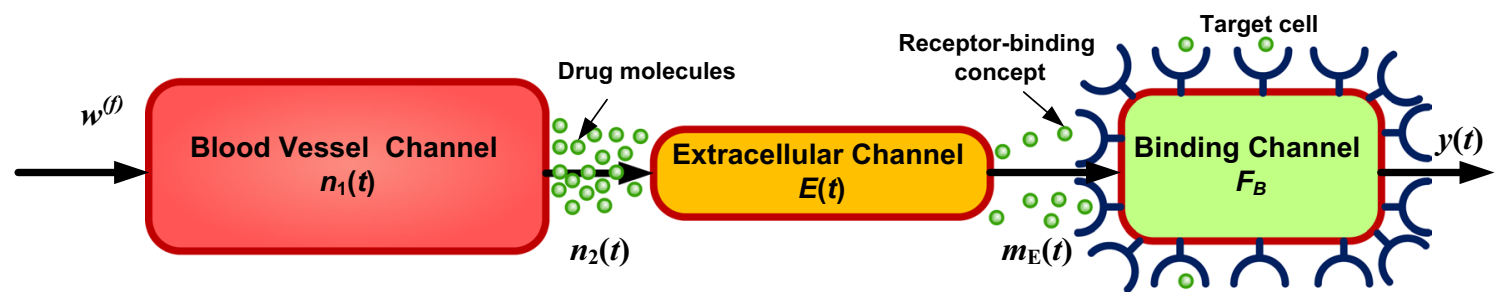

Fig. 4 The block diagram of the forward link, including binding channel

where $E(t)$ is the impulse response of the extracellular channel, and $n_{2}(t)$ denote the concentration of the drug molecules in the target site, while $*$ denotes the convolution operator.

Additionally, the movement of the molecules in the ECM channel may be explained as the difference between the tissues in the body, which is depending on the geometry, arrangement, tortuosity, and density. Therefore, the movement of the molecules varies in different parts of the body. We denote the diffusion coefficient around a blood vessel as $D_{E}$, while we represent the structure of the ECM with froth. The movement of the molecules is considered random motion; the molecules collide with the membrane of the cells. This affects the concentration distribution of these molecules. According to [32, 35], the characteristics of the tissues have the potential to infer an expression to $D_{E}$ by using the theory of transport in porous media. Practically, the transport or the movement in the ECM is dependent on the diffusion; thus, the transport by the interstitial pressure difference is ignored. The transport of the molecules by the diffusion coefficient in the ECM can be expressed in the function as follows [30]:

$E(t)=\frac{1}{\sqrt{2 \pi D_{E} t}} \exp \left(\frac{-z^{2}}{\left(4 D_{E}+k_{12}\right) t}\right)$

where $D_{E}$ is the diffusion coefficient of the diffusion channel in the ECM and $z$ is the counterpart to the target cell, and $k_{12}$ is the kinetic constant which represents the firstorder rate reaction between the drug molecules and other molecules. According to [1], the diffusion coefficient in the ECM depends on some parameters, as illustrated in the following equation:

$D_{E}=\frac{\phi}{k} D$

where $\Phi$ is the porosity that gauges the propensity of the tissues' component to pass the drug molecules to the target cell, denoting the tortuosity which is the arc length of the link between the input and output of the channel over geometric distance, following [15], the value of tortuosity $k$ must be high. Therefore, we considered the crowded proteinloaded environment into consideration and thus the value of $k=9$. Finally, the parameter $D$ is the diffusion coefficient medium that is used in Eq. (13).

\subsection{Binding Channel}

In the binding channel model, the receptors at the surface of the target cell have the capability of binding the molecules to come from the ECM. This binding is affected by the chemical affinity between the receptors at the surface of the target cells and the molecules from the ECM as well as the physical pressure resulting from the flow of these molecules in the ECM. As illustrated in Fig. 4, the function $F_{B}$ represents the receptors-binding channel, and the output $y(t)$ is the function of time that represents the concentration of the drug molecules bound to the targeted cells:

$y(t)=F_{B} \times m_{E}(t)$

The receptor-binding channel probability $F_{B}$ depends on some parameters, the total concentration of the drug molecules bound to the receptors on the surface of the target cell. As well as the ideal gas constant and the temperature of the patient as illustrated in the Section of numerical results.

\section{Numerical Results and Discussion}

In this section, we present the numerical results of the proposed framework-based IoBNT. The performance evaluation is considered for delivering the drug molecules to the diseased (targeted) cells, while ensuring there is no side effects on the healthy cells, and the reactiveness of these cells before binding to the drug molecules. The effectiveness of the proposed framework from the medical personnel to the diseased (targeted) cells is evaluated according to:

1. the porosity, $\Phi$ which is responsible for passing the drug molecules to the diseased cells,

2. the concentration, $\delta_{0}$ of the emitted molecules bound to the diseased cells, and

3. the effectiveness of some parameters such as $k_{12}$ and $k_{10}$ and the varying values of some parameters on the reverse and forward links. 
We campaign the simulation by using MATLAB for confirming our results. We use the default parameters for each plot as depicted in Table 2. Furthermore, for choosing the implemented parameters for each scenario, we used the previous experiments performed in $[1,2,29,30,48,49]$. The effectiveness parameters that will be used in the evaluation performance in this study are listed in Table 3:

\subsection{Effects of Drug Concentration in Forward Link}

The results in Fig. 5 illustrate the effects of the various parameter values on the concentration of the drug bound for the diseased (targeted) cells. We used (2), (3), (15), (16), and (18) to obtain these results. Figure 5a shows the varying values of porosity, which estimate the shape of the ECM, for allowing the drug molecules to pass through the blood capillary to the infected cells inside the target tissues. Furthermore, as shown in Fig. 5a, a higher porosity increases the concentration of drug molecules on the surface of the diseased cells, and the higher the binding by these cells inside the target tissues and at the same time the lower the binding probability by healthy cells within the same region. This leads to a rapid absorption by infected cells, increasing the plasma concentration, decreasing the latency and resulting in a fast elimination for the dose, and leading to a decrease in the duration of the effects.

By contrast, the effects of the varying values of the emitted molecules from the bio-cyber interface encapsulated by liposome and spread within the blood vessel network are as shown in Fig. 5b. Moreover, it can be seen in Fig. 5b that a higher concentration of emitted molecules leads to an increase in the concentration of the drug molecules around the reception space of the diseased cells inside the target nanonetwork (target tissue). This means the emitted molecules efficiently reach Nano-R2 as drug molecules and

Table 3 Default simulation parameter

\begin{tabular}{ll}
\hline Parameters & Value \\
\hline$\Phi$ & 0.5 \\
$m_{0}$ & $5 \mu \mathrm{m}$ \\
$k_{12}$ & $0.373 \mathrm{~min}^{-1}$ \\
$k_{10}$ & $0.172 \mathrm{~min}^{-1}$ \\
$\delta_{0}$ & $0.7 \mathrm{Ml}$ \\
$T$ & $37^{\circ} \mathrm{C}$ \\
$\xi$ & 1 \\
$r_{R}$ & $60 \mathrm{~nm}$ \\
$R$ & $10 \mu \mathrm{m}$ \\
$k_{21, r}$ & $0.073 \mathrm{~min}^{-1}$ \\
$k_{L}$ & $0.001 \mathrm{~min}^{-1}$ \\
$\alpha_{\mathrm{m}}$ & $15 \mu \mathrm{M}$ \\
$X$ & $40 \mu \mathrm{L}$ \\
\hline
\end{tabular}

increase their binding with the diseased cells, reaching Nano-R3 as information molecules for eliminating the dose.

Figure 5c shows how a higher constant forward rate $k_{12}$ leads to an increase in the concentration of the drug molecules around the reception space inside the target nanonetwork (target tissues). Furthermore, Fig. 5c shows that this parameter depends on the difference in concentration between the compartments, as well as the fenestra size through the endothelial cell network and based on the diffusion properties of the information molecules. It does not have control over this parameter.

We can determine the effects of the variation in the elimination rate, $k_{10}$, in Fig. 5d. Based on this figure, it can be seen that $k_{10}$ impacts both the concentration of the drug molecules bound to the infected cells and the IoBNT. This implies that a higher value of this parameter leads to a rapid elimination, decreasing both the peak of the plasma concentration and the duration of the effects.

Figure 5e, illustrates the effects of single and multiple liposomes carriers of the drug molecules in the concentration of drug around the diseased cell inside targeted intrabody nanonetwork. We used in our proposed model one liposome as a default, but as shown in Fig. 5e by increasing the number of liposomes, it gives a high concentration of the drug molecules which bounded to the receptors of the surface in the reception space around diseased cell. Moreover, it achieved to improve the therapeutic drug by modifying drug absorption and reducing the toxicity around healthy cells. It also, helped to control the drug distribution by properties of the liposomes which bounded to the diseased cell. So, it leads to there is no effect of any physicochemical characteristics of the drug in intra-body nanonetwork.

\subsection{Normalized Concentration in Reverse Link}

We employed (7), (8), (9), (10), and (14) to obtain the results in Fig. 6. The results indicate the effects of the varying parameter values on the normalization of the bioluminescence intensity. Figure 6a shows the effects of the different values of parameter $r_{R}$, which is the radius of Nano-R2, indicating the reception space around the diseased cells inside the targeted nanonetwork. The plot indicates that increasing the surface area results in a higher concentration of the drug molecules around the infected cells. This means that increasing the activated molecules binding to the receptors on the surface of Nano-R2 decreases the latency and the side effects around the healthy cells at the same site. Moreover, in this study we consider the size of the fenestrae in the ECM for transporting the drug molecules to the infected cells; therefore, we set the maximum radius of Nano-R2 to $150 \mathrm{~nm}$ as the default.

The plot in Fig. 6b shows that the short distance between the nanotransmitter Nano-R1 and the nanoreceiver Nano-R2 

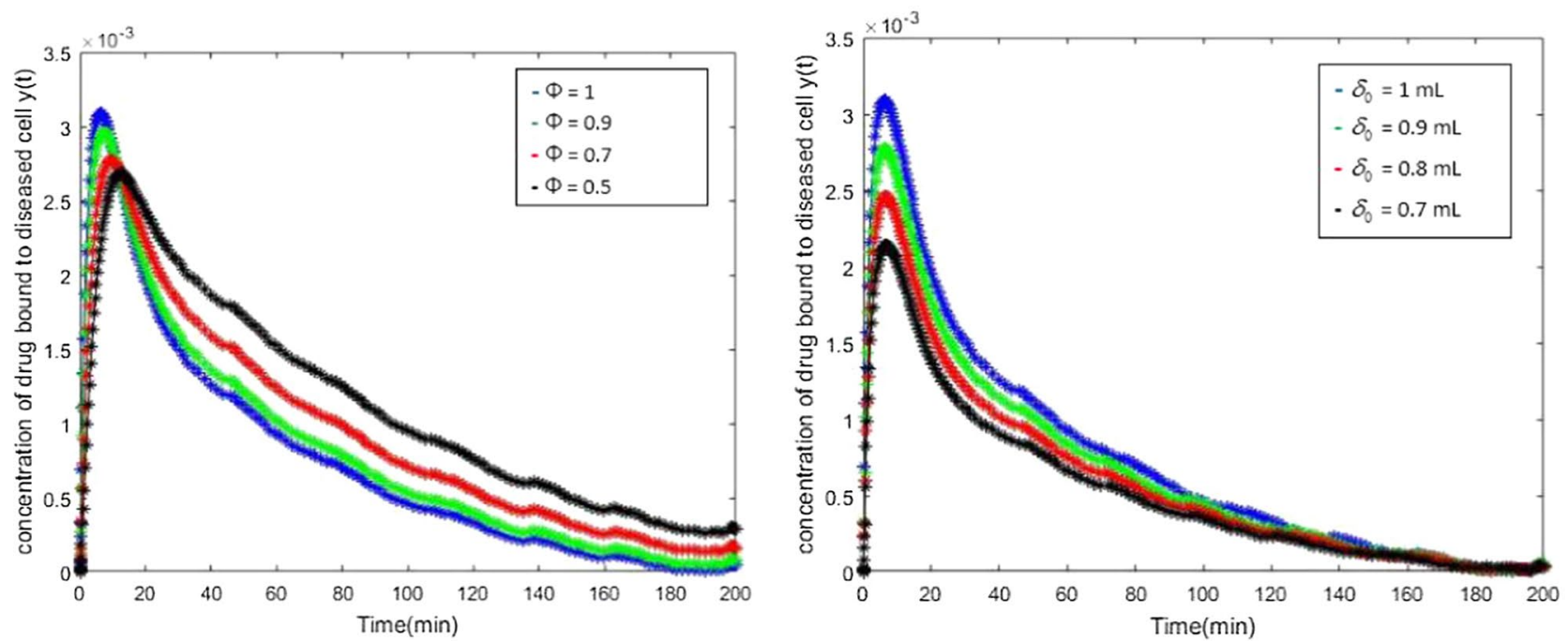

(a)

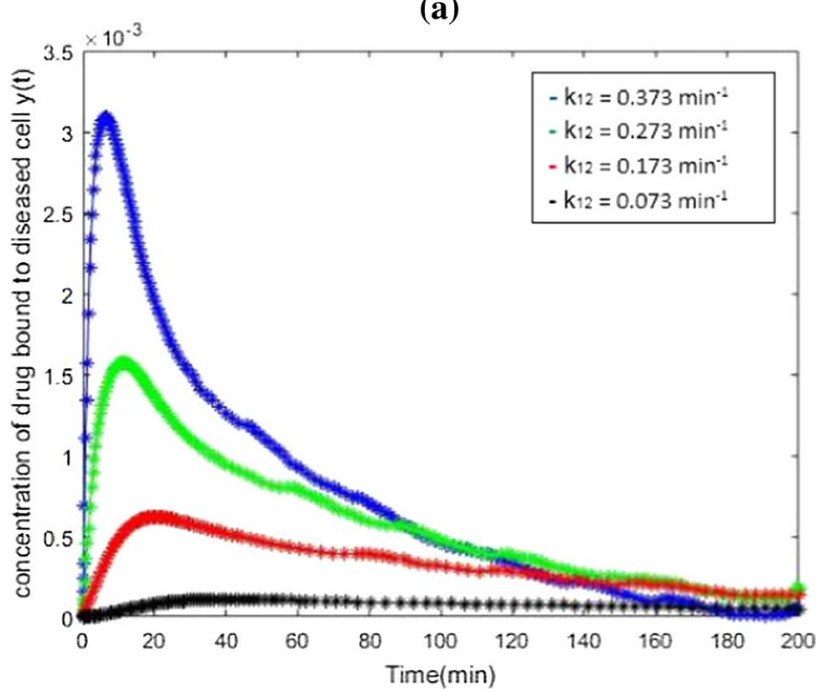

(c)

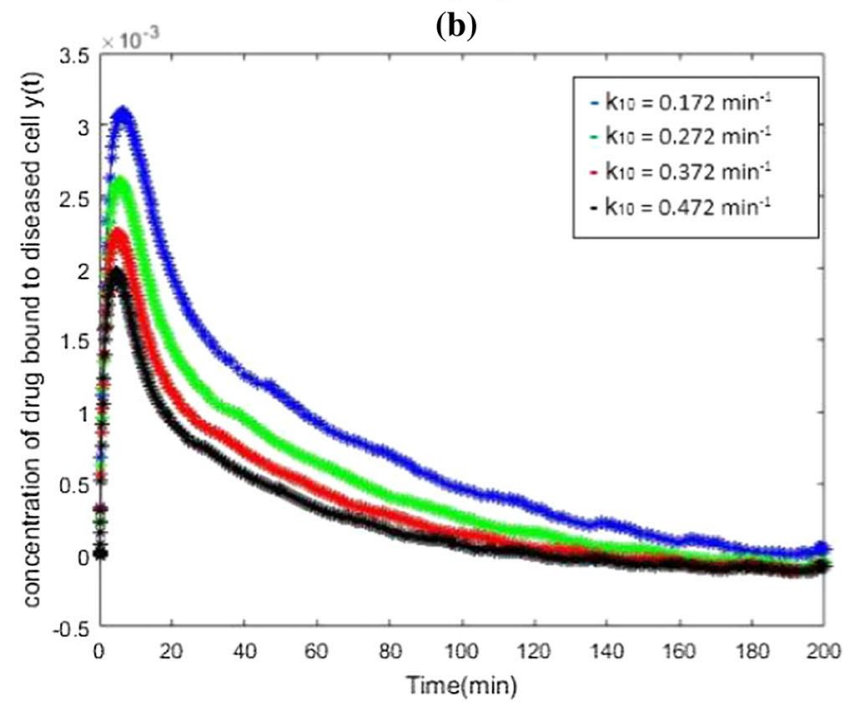

(d)

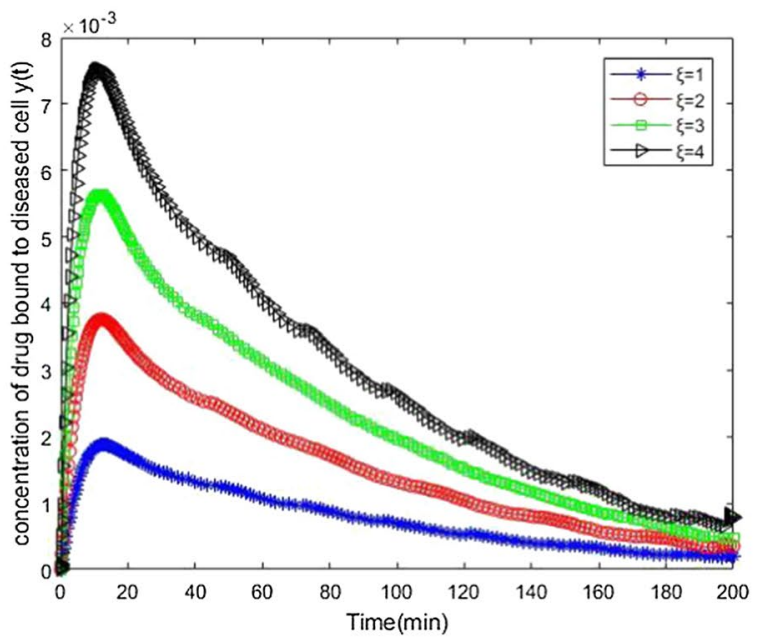

(e)

Fig. 5 The variance of drug concentration in the forward link 


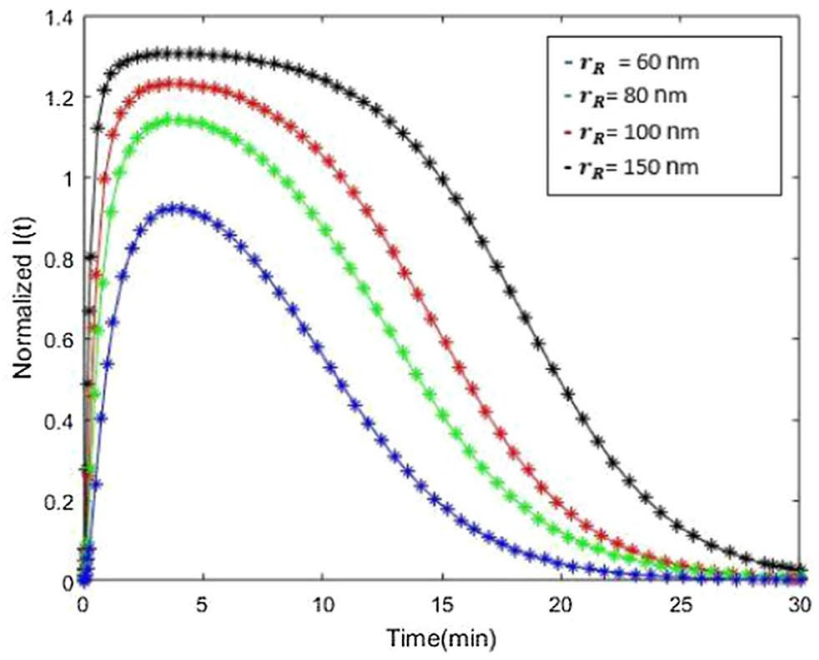

(a)

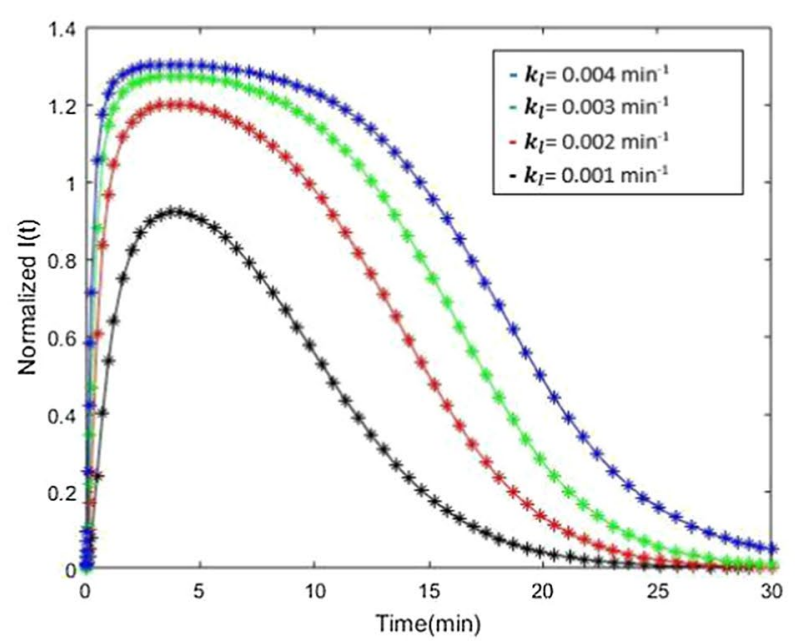

(c)

Fig. 6 The variance of normalized concentration in the reverse Link

leads to more rate diffusion from the high intensity of the drug molecules around Nano-R1 to the low intensity of the region around Nano-R2. This means that increasing the peak of the plasma concentration around the infected cells also increases the uptake of the drug molecules through the receptors on the surface of Nano-R2, and that there are no free drug molecules binding to the healthy cells. By contrast, the large distance between Nano-R1 and Nano-R2 decreases the plasma concentration around diseased cells, leading to more free drug molecules binding with healthy cells at the same site. It is therefore better to keep the distance short to decrease side effects around healthy cells.

As shown in Fig. 6c, the higher values of $k_{L}$ increase the normalized concentration; thus, the bio-cyber interface becomes more efficient at detecting the information molecules. This parameter depends on the distribution of

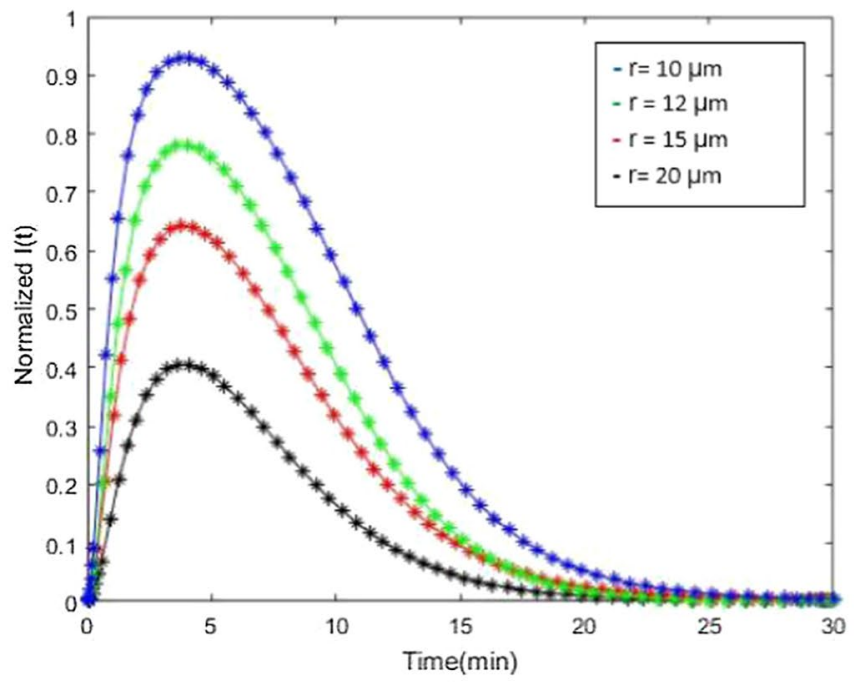

(b)

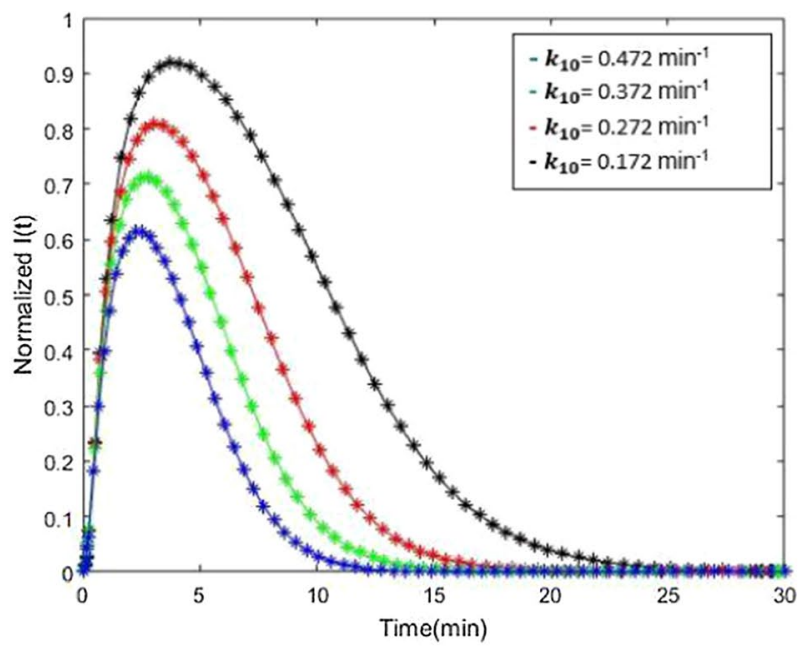

(d)

the molecules outside the vascular space and entering the bio-cyber interface. It also depends on the concentration of the receptors, which means the size and number of information molecules.

Figure $6 \mathrm{~d}$ shows the effects of the values of the elimination rate $k_{10}$; this parameter influences both the performance of IoBNT and the bioluminescence intensity, and measures the loss of the information molecules resulting from the reaction process, adhesion process, elimination by the liver, and absorption through non-targeted cells. Therefore, the higher the value of this parameter, the higher the bioluminescence intensity. As mentioned above, increasing the value of this parameter leads to a fast elimination of the dose and a decrease in the duration of the effects. 


\subsection{Effects of the Binding Parameter on the Concentration of the Drug to the Diseased Cells}

As described above, the binding parameter $\mathrm{F}_{\mathrm{B}}$ represents the relationship between the molecules around the target cell and the molecules bound to the receptors at the surface of the target cell. Furthermore, we employed an equation mimicking $\mathrm{F}_{\mathrm{B}}$ as follows [10]:

$F_{B}=\frac{m_{0}}{R T} k_{12}$.

Figure 7 shows the effects of the values of certain parameters on the concentration of the drug molecules. As illustrated in Fig. 7a, a higher value of $m_{0}$ leads to an increase in the value of the binding parameter. Thus, we demonstrated that increasing the concentration of the drug molecules around the target cell leads to an increased binding of the receptors at the surface of the target cell, all of which leads to an increased absorption by the diseased cells and a minimal loss of drug molecules, along with a decrease in the side effects. Figure $7 \mathrm{~b}$ shows that increasing the value of $k_{12}$ increases the sizes of the endothelial cells, which separates the target BAN from the blood vessel. This means that increasing the diffusion of the drug molecules around an infected cell can lead to an increased binding of the receptors at the surface of the target, or diseased, cell.

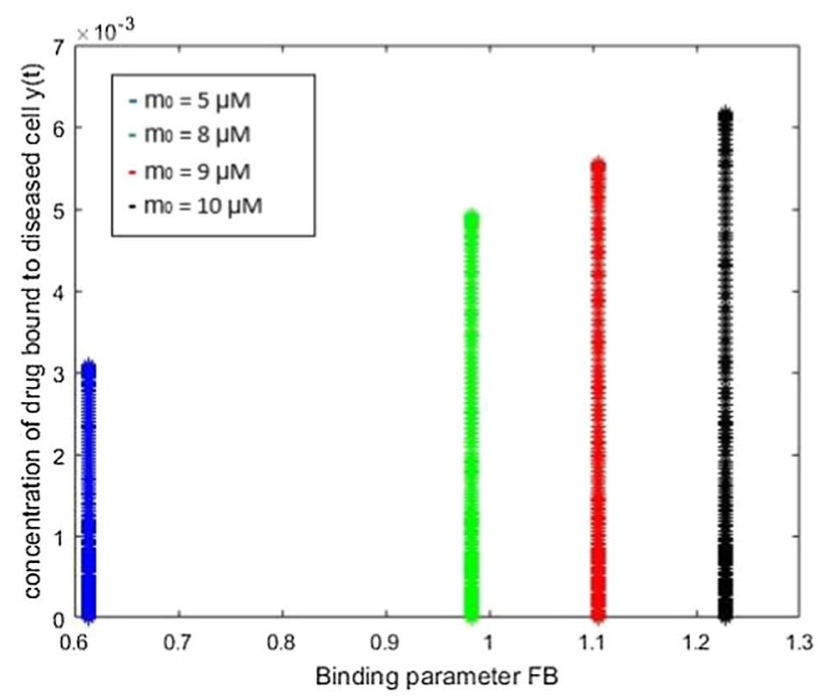

(a)

\subsection{Performance Comparison}

In this section, we present the performance comparison between our proposed model and the previous work which verifies the same behavior of the proposed model in forward and reverse link. Additionally, we study the influence of system's parameters on the performance as follows.

\section{- Forward link}

Figure 8a shows a performance comparison based on the concentration of the drug molecules for the forward link between our proposed model and previous studies. The simulation parameters of this comparison are listed inside the figure. As we can see, the proposed model verifies the same performance as in previous work; however, the proposed model is significantly superior, achieving a higher concentration for drug molecules spread from a bio-cyber interface to the target site. In addition, the proposed model is capable of preventing the occurrence highly localized and quickly growing diseased cells by taking into account the target site, the distance between the nanotransmitter and nanoreceiver, and the binding receptors around the nanoreceiver space. Moreover, the duration time of the drug molecules in the blood vessel network until reaching the infected cells in the target site through the endothelial cells is reduced, because the proposed model considers the porosity of the endothelial cells. Subsequently, the proposed model can decrease the side effects around healthy cells during target drug delivery and achieve a good plasma concentration when compared with the previous approach.

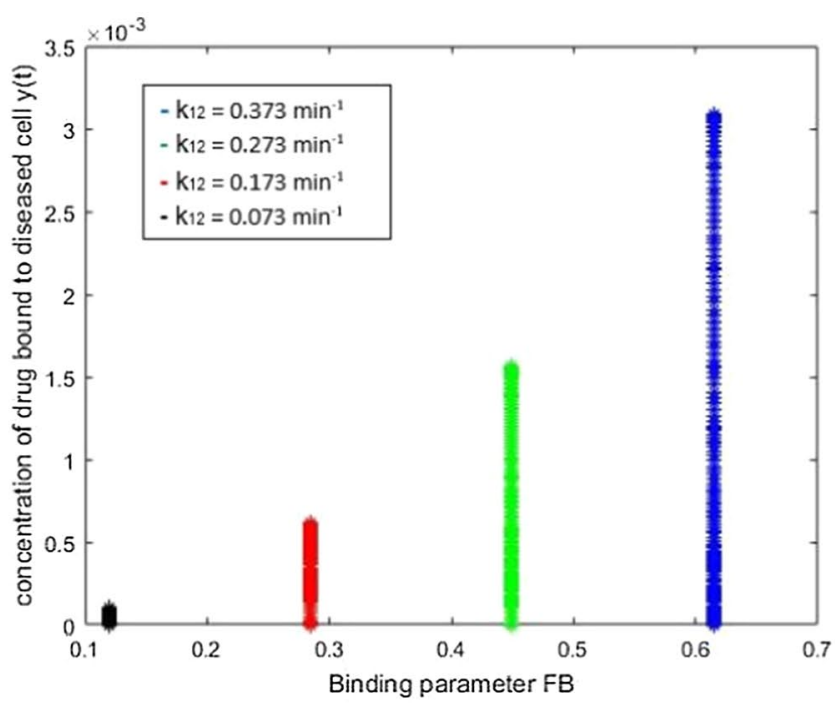

(b)

Fig. 7 Effects of binding parameters on the concentration of drug 


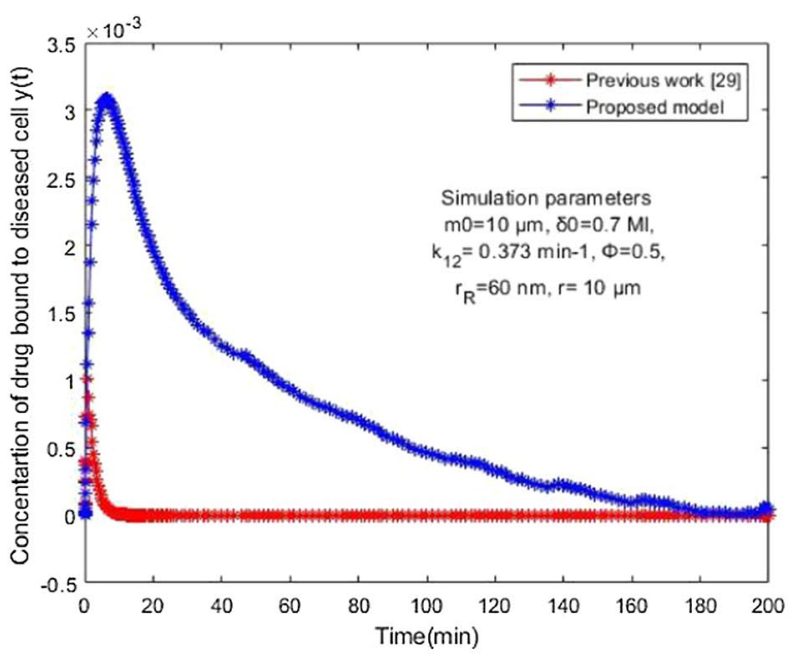

(a)

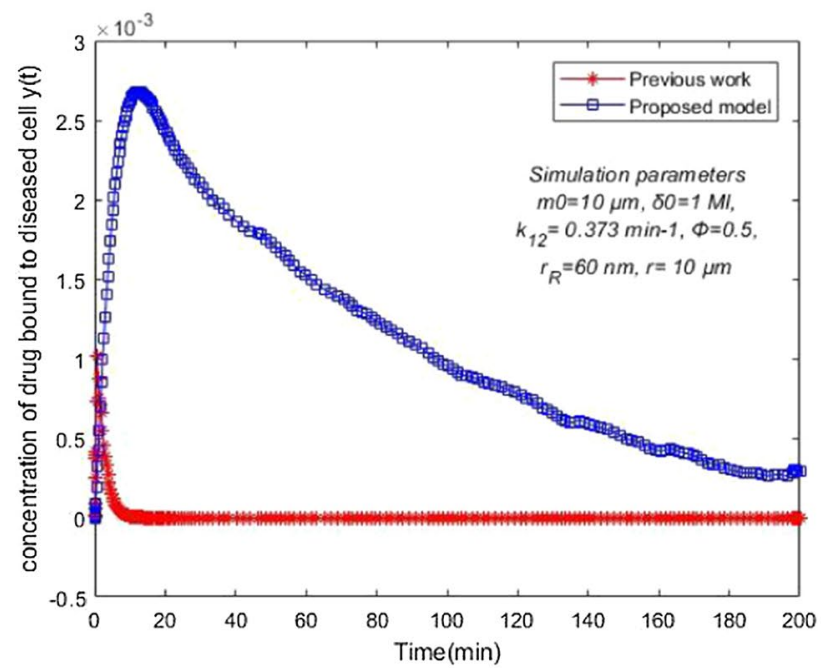

(b)

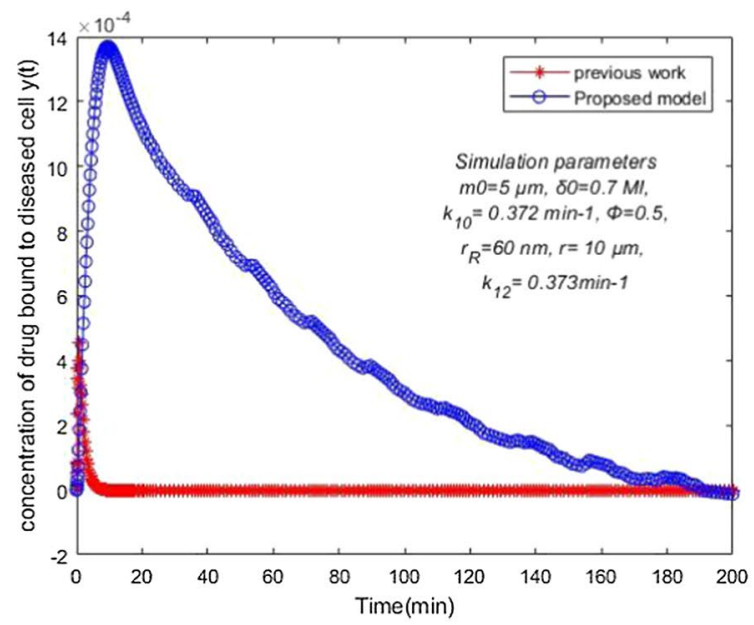

(c)

Fig. 8 Performance comparison between the proposed model and the previous work in forward link
Figure $8 \mathrm{~b}$ shows the performance comparison of the bioluminescence intensity received by the reception space around the infected cells in a reverse link for the proposed model and previous study. As shown, the proposed model clearly achieves high bioluminescence intensity for the molecules around the reception space of the diseased cells by considering the radius of the reception space as compared with the previous method. Because the proposed model can achieve a fast absorption by applying a high affinity of the receptors on the surface of the targeted cells as well as it can decrease the side effects of the targeted drug delivery in the forward and reverse links as compared with the previous approach.

Figure 8c illustrates the influences of $k_{10}$ on the concentration of the drug molecules which bounded to the targeted cells. Obviously, increasing the value of $k_{10}$ leads to fast elimination process between nanodevices in the previous work wherein it is looked that there is no more drug molecules concentration around diseased cells as shown the peak of plasma concentration of red curve. In contrast, the proposed model exploits the increasing of $k_{10}$ and thus providing a good concentration of the drug molecules at the reception space around diseased cells. Furthermore, the proposed model provides an efficient communication between nanodevices in the targeted nanonetwork but not finishing the elimination process between these nanodevices until ensure that most of the drug molecules bounded by the ligand-receptors at the reception space around diseased cells.

\section{- Reverse link}

Figure 9 illustrates the performance comparison of the bioluminescence intensity received by the reception space around infected cells in reverse link for the proposed model and pervious work. As we can observe, the proposed model has high bioluminescence intensity for molecules around the reception space of diseased cells by taking into consideration the radius of the reception space comparing with the previous work. Owning to the proposed model can achieve fast absorption by applying the high affinity of the receptors on the surface of the targeted cells. Therefore, the proposed model based on IoBNT can decrease the side effects of targeted drug delivery in forward and reverse link comparing with the previous work.

When compared the proposed model with the previous work [29], we could conclude that, the proposed framework takes into account the physical interactions of the receptor proteins with the received ligand as well as the characteristic parameters of the targeted receptors. Subsequently, it can achieve a fast absorption, reduce the 


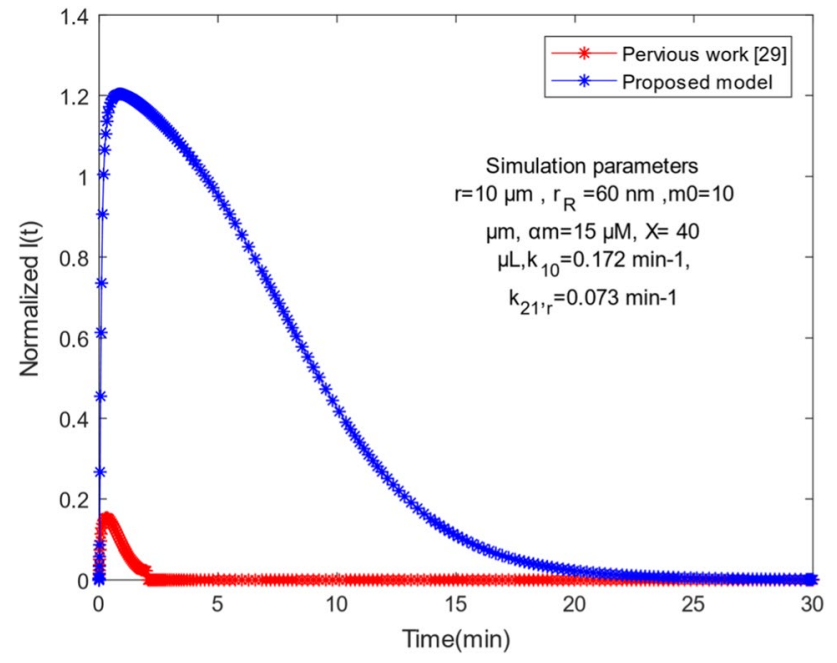

Fig. 9 Performance comparison between the proposed model and previous work in reverse link

duration time of the dose advection and diffusion in the blood stream, and thus reduce the side effects. In other words, the drug molecules precisely locate the diseased cells without affecting the healthy cells. In addition, it prevents highly localized and quickly growing diseased cells. Therefore, the proposed framework based on IoBNT can be considered as a promising step toward the engineering of a targeted drug delivery system.

\section{Conclusion}

We presented a framework-analysis-based compartmental model of IoBNT for application to the healthcare industry. We studied the effects and variances of a drug concentration around diseased (targeted) cells inside an intra-body nanonetwork. We also modeled a BAN using a high-affinity ligand to increase the binding with the receptors at the surface of the target cell. The effects of the system and the binding parameter on the performance of the IoBNT were analyzed. The analysis and numerical results of the proposed IoBNT were verified through a simulation. The results indicate that the developed compartmental-model-based IoBNT enhances the delivery of drug molecules to diseased cells with minimal side effects when compared with a previous model.

\section{References}

1. Chude-Okonkwo, U.A.; Malekian, R.; Maharaj, B.S.: Molecular communication model for targeted drug delivery in multiple disease sites with diversely expressed enzymes. IEEE Trans. Nanobiosci. 15(3), 230-245 (2016)
2. Chude-Okonkwo, U.A.; et al.: Bio-inspired approach for eliminating redundant nanodevices in Internet of Bio-nano things. In: 2015 IEEE Globecom Workshops (GC Wkshps). IEEE (2015)

3. Akyildiz, I.F.; Brunetti, F.; Blázquez, C.: Nanonetworks: a new communication paradigm. Comput. Netw. 52(12), 2260-2279 (2008)

4. Nistor, M.T.; Rusu, A.G.: Nanorobots with applications in medicine. In: Polymeric Nanomaterials in Nanotherapeutics, pp. 123149. Elsevier (2019)

5. Singh, A.; Bivalacqua, T.J.; Sopko, N.: Urinary tissue engineering: challenges and opportunities. Sexual Med. Rev. 6(1), 35-44 (2018)

6. Hoekstra, R.; Blondeau, P.; Andrade, F.J.: Distributed electrochemical sensors: recent advances and barriers to market adoption. Anal. Bioanal. Chem. 410(17), 4077-4089 (2018)

7. Akyildiz, I.F., et al.: The internet of-nano things. IEEE Commun. Mag. 53(3), 32-40 (2015)

8. Chahibi, Y.; Pierobon, M.; Akyildiz, I.F.: Pharmacokinetic modeling and biodistribution estimation through the molecular communication paradigm. IEEE Trans. Biomed. Eng. 62(10), 2410-2420 (2015)

9. Atakan, B.; Akan, O.B.; Balasubramaniam, S.: Body area nanonetworks with molecular communications in nanomedicine. IEEE Commun. Mag. 50(1), 28-34 (2012)

10. Youssef, C., et al.: A molecular communication system model for particulate drug delivery systems. IEEE Trans. Biomed. Eng. 60(12), 3468-3483 (2013)

11. Cai, P., et al.: Combinatorial nano-bio interfaces. ACS Nano 12(6), 5078-5084 (2018)

12. Nakano, T.; et al.: Swarming biological nanomachines through molecular communication for targeted drug delivery. In: Proceedings of the SCIS-ISIS. (2012)

13. Nakano, T., et al.: Molecular communication among biological nanomachines: a layered architecture and research issues. IEEE Trans. Nanobiosci. 13(3), 169-197 (2014)

14. Abd El-atty, S.M.; Bidar, R.; El-Rabaie, S.: MolCom system with downlink/uplink biocyber interface for internet of Bio-NanoThings. Int. J. Commun. Syst. 23(1), 1-21 (2020)

15. El-atty, S.M.A.; El-Taweel, A.; El-Rabaie, S.: Transmission of nanoscale information-based neural communication-aware ligand-receptor interactions. Neural Comput. Appl. 30(11), 3509-3522 (2018)

16. El-Fatyany, A.; Wang, H.; Abd El-atty, S.M.: On mixing reservoir targeted drug delivery Modeling-based Internet of Bio-NanoThings. Springer Wirel. Netw. 26(5), 3701-3713 (2020)

17. El-Fatyany, A., et al.: Biocyber interface-based privacy for internet of bio-nano things. Wirel. Pers. Commun. 114, 1465-1483 (2020)

18. Abd El-atty, S.M.: Health monitoring scheme-based FRET nanocommunications in internet of biological nanothings. Int. J. Commun. Syst. 33(11), 1-17 (2020)

19. Abd El-atty, S.M.; Tolba, A.: A cross-layer approach for optimization of molcom systems toward the internet of bio-nanothings. IEEE Syst. J. 13(3), 2751-2762 (2018)

20. El-atty, S.M.A., et al.: Engineering molecular communications integrated with carbon nanotubes in neural sensor nanonetworks. IET Nanobiotechnol. 12(2), 201-210 (2017)

21. Chen, M., et al.: Body area networks: a survey. Mobile Netw. Appl. 16, 661-662 (2011)

22. Nakano, T., et al.: Molecular communication and networking: opportunities and challenges. IEEE Trans. Nanobiosci. 11(2), 135-148 (2012)

23. Felicetti, L., et al.: TCP-like molecular communications. IEEE J. Sel. Areas Commun. 32(12), 2354-2367 (2014) 
24. Femminella, M.; Reali, G.; Vasilakos, A.V.: A molecular communications model for drug delivery. IEEE Trans. Nanobiosci. 14(8), 935-945 (2015)

25. Chude-Okonkwo, U.A., et al.: Molecular communication and nanonetwork for targeted drug delivery: a survey. IEEE Commun. Surv. Tutorials 19(4), 3046-3096 (2017)

26. Chude-Okonkwo, U.A., et al.: Information-theoretic model and analysis of molecular signaling in targeted drug delivery. IEEE Trans. Nanobiosci. 19(2), 270-284 (2020)

27. Loscri, V., et al.: Security and privacy in molecular communication and networking: opportunities and challenges. IEEE Trans. Nanobiosci. 13(3), 198-207 (2014)

28. Felicetti, L., et al.: Applications of molecular communications to medicine: a survey. Nano Commun. Netw. 7, 27-45 (2016)

29. Chude-Okonkwo, U.A.; Malekian, R.; Maharaj, B.: Biologically inspired bio-cyber interface architecture and model for Internet of bio-nanothings applications. IEEE Trans. Commun. 64(8), 3444-3455 (2016)

30. Chahibi, Y., et al.: Molecular communication modeling of antibody-mediated drug delivery systems. IEEE Trans. Biomed. Eng. 62(7), 1683-1695 (2015)

31. Deshpande, P.P.; Biswas, S.; Torchilin, V.P.: Current trends in the use of liposomes for tumor targeting. Nanomedicine $\mathbf{8}(9)$, 1509-1528 (2013)

32. Olusanya, T., et al.: Liposomal drug delivery systems and anticancer drugs. Molecules 23(4), 907 (2018)

33. Di Paolo, D., et al.: Drug delivery systems: application of liposomal anti-tumor agents to neuroectodermal cancer treatment. Tumori J. 94(2), 246-253 (2008)

34. Dollard, M.-A.; Billard, P.: Whole-cell bacterial sensors for the monitoring of phosphate bioavailability. J. Microbiol. Methods 55(1), 221-229 (2003)

35. Monson, E., et al.: PEBBLE nanosensors for in vitro bioanalysis. Biomed. Photon. Handb. 9, 1-14 (2003)

36. Miah, M.K.; et al.: Clinical pharmacokinetics. In: Clinical Pharmacy Education, Practice and Research, pp. 409-424. Elsevier (2019)

37. ShahMohammadian, H.; Messier, G.G.; Magierowski, S.: Optimum receiver for molecule shift keying modulation in diffusionbased molecular communication channels. Nano Commun. Netw. 3(3), 183-195 (2012)
38. Klein, B.G.: Cunningham's Textbook of Veterinary Physiology. Elsevier Health Sciences, New York (2019)

39. Close, D.M.; et al.: Light without substrate amendment: the bacterial luciferase gene cassette as a mammalian bioreporter. In: Sensing Technologies for Global Health, Military Medicine, Disaster Response, and Environmental Monitoring; and Biometric Technology for Human Identification VIII. International Society for Optics and Photonics (2011)

40. Gutierrez, P.S.; Monteoliva, D.; Diambra, L.: Cooperative binding of transcription factors promotes bimodal gene expression response. PLoS ONE 7(9), e44812 (2012)

41. Tyler, J.; Shiu, A.; Walton, J.: Revisiting a synthetic intracellular regulatory network that exhibits oscillations. J. Math. Biol. 78(7), 2341-2368 (2019)

42. Weiss, R., et al.: Genetic circuit building blocks for cellular computation, communications, and signal processing. Nat. Comput. 2(1), 47-84 (2003)

43. $\mathrm{Xu}, \mathrm{T}$., et al.: Improving estrogenic compound screening efficiency by using self-modulating, continuously bioluminescent human cell bioreporters expressing a synthetic luciferase. Toxicol. Sci. 168(2), 551-560 (2019)

44. Bonate, P.: Advanced methods of pharmacokinetic and pharmacodynamic systems analysis. IDrugs Investigation. Drugs J. 4(9), 1017-1020 (2001)

45. Contag, C.H.; Bachmann, M.H.: Advances in in vivo bioluminescence imaging of gene expression. Annu. Rev. Biomed. Eng. 4(1), 235-260 (2002)

46. Marques, S.M.; da Silva, J.C.E.: Firefly bioluminescence: a mechanistic approach of luciferase catalyzed reactions. IUBMB Life 61(1), 6-17 (2009)

47. Yan, Y., et al.: Firefly-inspired approach to develop new chemiluminescence materials. iScience 13, 478-487 (2019)

48. Hamad, H.T; Abbood, D.W.; Mustafa, A.S.: Strength tortuosityporosity relation in locally types of porous media (experimental model). In: IOP Conference Series: Materials Science and Engineering. IOP Publishing (2018)

49. Matyka, M.; Khalili, A.; Koza, Z.: Tortuosity-porosity relation in porous media flow. Phys. Rev. E 78(2), 026306 (2008) 